\title{
Geotail observation of counter directed ESWs associated with the separatrix of magnetic reconnection in the near-Earth magnetotail
}

\author{
S. Y. Li ${ }^{1,2}$ Y. Omura, ${ }^{1}$ B. Lembège, ${ }^{1,3}$ X. H. Deng, ${ }^{4}$ H. Kojima, ${ }^{1}$ Y. Saito, ${ }^{5}$ \\ and S. F. Zhang ${ }^{2}$ \\ Received 15 April 2013; revised 19 November 2013; accepted 9 December 2013; published 8 January 2014.
}

[1] A sub-Alfve'nic jet in the tailward outflow region near the separatrix of the magnetic reconnection is observed by Geotail on 9 February 1995. Several dozens of electrostatic solitary waves/pulses (ESWs) are observed, respectively, on the current sheet-side and the lobe-side of the separatrix. The ESWs on the current sheet-side are of type-B with direction outward (toward to the tailward) while on the lobe-side they are of type-A directed to X-line. The amplitude of ESWs on the current sheet-side is about 6 times more than those on the lobe-side, suggesting that energies flowing outward from the reconnection X-line are much larger than those flowing inward. Moreover, observations show, that electron beams associated with ESWs, which are parallel to the ambient magnetic field, are much stronger on the current sheet-side than on the lobe-side of the separatrix. Furthermore, the direction of the electron beam on the lobe-side of the separatrix is mainly antiparallel to the ambient magnetic field and it is mainly parallel on the current sheet-side. Both are consistent with the propagation of ESWs which is in agreement with the generation mechanism of ESWs, which is suggested to be related to electron beams. These results are helpful for solving the issue of ESWs' generation mechanism associated with magnetic reconnection. It also provides an important clue for understanding the fast energy release during the magnetic reconnection process.

Citation: Li, S. Y., Y. Omura, B. Lembège, X. H. Deng, H. Kojima, Y. Saito, and S. F. Zhang (2014), Geotail observation of counter directed ESWs associated with the separatrix of magnetic reconnection in the near-Earth magnetotail, J. Geophys. Res. Space Physics, 119, 202-210, doi:10.1002/2013JA018920.

\section{Introduction}

[2] The magnetic reconnection is a key mechanism invoked for the energy release and transformation from the previously stored magnetic energy to the kinetic energy of particles by changing the topology of magnetic field line in a local region and has been widely studied for tens of years. The magnetic separatrix is an important boundary layer separating the inflow and outflow regions in magnetic reconnection. The separatrix region can maintain its internal structure over at least several hundreds of ion skin depths [André et al., 2004; Retinò

\footnotetext{
${ }^{1}$ Research Institute for Sustainable Humanosphere, Kyoto University, Uji, Kyoto, Japan.

${ }^{2}$ College of Aerospace Science and Engineering, National University of Defense Technology, Changsha, China.

${ }^{3}$ LATMOS_IPSL_UVSQ_CNRS, Guyancourt, France.

${ }^{4}$ Nanchang University, Nanchang, China.

${ }^{5}$ Institute of Space and Astronautical Sciences, Japan Aerospace Exploration Agency, Sagamihara, Kanagawa, Japan.

Corresponding author: S. Y. Li, College of Aerospace Science and Engineering, National University of Defense Technology, Changsha, 410073, China. (li.shiyou.qiu@gmail.com)

(C2013. American Geophysical Union. All Rights Reserved. 2169-9380/14/10.1002/2013JA018920
}

et al., 2006; Khotyaintsev et al., 2006]. It is a rather dynamic region with wave-particle interactions. During the crossing of the separatrix with the reversal of plasma flow and Hall magnetic fields, strong electrostatic lower-hybrid-drift (LHD) mode was observed [Wang et al., 2010].

[3] The electrostatic solitary waves (ESWs) in the space are regarded to be the electron phase-space potential holes (electron holes) which are Bernstein-Greene-Kruskal (BGK) modes, i.e., one-dimensional equilibrium solutions to the time-independent Vlasov-Poisson equations [Bernstein et al., 1957; Chen and Parks, 2002]. ESWs have been studied for many years since they were regarded as a kind of Broadband Electrostatic Noise (BEN) in 1990s [Matsumoto et al., 1994a; Kojima et al., 1994]. They have been observed in many key space regions, including the solar wind, the upstream and downstream regions of the terrestrial bow-shock, the magnetosheath and the magnetopause, the cusp region and the auroral acceleration region, the plasma sheet boundary layer, and the magnetotail [Matsumoto et al., 1994a, 1999, 2003; Kojima et al., 1997, 1999a; Mozer et al., 1997; Ergun et al., 1998; Bale et al., 1998; Cattell et al., 1999, 2003; Pickett et al., 2003, 2004, 2005; Shin et al., 2008]. In the magnetotail, numerous ESWs have been observed in the plasma sheet boundary layer (PSBL) and their characteristics have also been analyzed [Matsumoto et al., 1994a, 1999; 
Kojima et al., 1997, 1999a]. The propagation of ESWs has also been studied in the boundary layers [Pickett et al., 2004, 2007].

[4] The ESWs have also been observed in/around the magnetic reconnection diffusion region (hereafter called MRDR) both by in situ observations [Deng et al., 2004, 2009; Cattell et al., 2005; Li et al., 2009, 2010, 2013a, 2013b] and by simulations [Drake et al., 2003; Cattell et al., 2005; Lapenta et al., 2011]. The observation evidence of high-frequency electrostatic waves inside an ion-scale magnetic flux rope embedded in a reconnecting current sheet in the Earth's magnetotail was also reported [Khotyaintsev et al., 2010]. Some authors suggested that ESWs can contribute to accelerating electrons in the reconnection process [e.g., Farrell et al., 2002; Drake et al., 2003]. However, it was argued by some others, claiming that the ESWs are just some small spatial bubbles within the relatively larger reconnection region [Omura et al., 1996]. But it was accepted by both arguers that the ESWs around the reconnection diffusion region are mainly observed on the boundary layer of the reconnection region and will be taken account to the change of the electron phase distribution.

[5] With respect to the study of ESWs associated with magnetic reconnection, one important issue is the generation source region of the ESWs and their role in the energy releasing process of magnetic reconnection. It is generally known that in the reconnection process, the electrons will decouple from the ions and be accelerated into a speed exceeding the Alfvenic velocity. The relative drift of electrons and ions or between different electron populations can then largely exceed the electron thermal speed and streaming instabilities, including the bi-streaming instability and bump-on-tail instability. These kinds of instability develop and then generate bipolar structures in electric field which was termed as electrostatic solitary waves. Particle-in-cell (PIC) simulation to the electron behavior in the reconnection with a guide field suggested that the ESWs can be generated near the magnetic X-line [Drake et al., 2003]. Cattell et al. [2005] suggested that the electron holes (i.e., ESWs) are excited in the separatrix region, near the outer edge of the current sheet. The electron holes occur during intervals when there were narrow electron beams, either unidirectional or counterstreaming. Recently, Lapenta et al. [2011] had also discussed the generation of ESWs in the reconnection process. They conducted massively parallel simulations for realistic conditions and for the hydrogen mass ratio in boxes larger than considered in similar previous studies. In all cases of their simulation result, along the separatrices a strong electron flow is observed, sufficient to lead to the onset of streaming instabilities and to form bipolar parallel electric field signatures.

[6] However, direct observations and analysis of ESWs in both sides of separatrix associated with magnetic reconnection are rarely reported. This paper mainly focuses on this issue and tries to answer the following questions: what kinds of ESWs can be observed on both sides of the separatrix? Are they locally generated? What are their respective propagating directions?

[7] In this paper, a magnetic reconnection event, which is with sub-Alfve' nic jet in the tailward outflow region near the separatrix, is to be studied. The ESWs are observed when the spacecraft skims the separatrix. The spatial structure of ESWs and their polarization will be analyzed. The relationship between the direction of ESWs and the electron beam is also investigated. High time-resolution data of the electric field from the Wave-Form Capture (WFC) instrument [Matsumoto et al., 1994b] are mainly employed for the analysis of the ESWs. The WFC serves as a part of the Plasma Waves Instrument (PWI). Three-dimensional electron and ion distribution data obtained from the Low Energy Particle (LEP) experiment [Mukai et al., 1994] are used to analyze the particle characteristics. Other data used herein include magnetic field data obtained from the Magnetic Field (MGF) experiment [Kokubun et al., 1994] and plasma moment data obtained from the LEP instrument.

[8] This paper is arranged as follows: in section 2, the magnetic reconnection event and the separatrix crossing are briefly addressed. The observation and analysis of ESWs associated with the separatrix are shown in section 3. In section 4, particle activities are shown and discussed. The discussion and concluding remarks will be presented in the last section.

\section{Overview of Magnetic Reconnection and Separatrix Crossing}

[9] A case of magnetic reconnection with long time duration has been encountered by Geotail from 09:55 to 13:10 UT on 9 February 1995, when the satellite is located in the nearearth tail current sheet at $(-29.85,2.06-0.26,-2.49) R_{E}$. This event has been studied in the previous work [ $\mathrm{Li}$ et al., 2012] and it will be addressed briefly herein.

[10] Figure 1 gives an overview of the observation history for the event with panels $(\mathrm{a}-\mathrm{b})$ plotting data from 09:40 UT to 11:20 UT and panels (c-g) showing data zoomed-in within interval of 10:20-11:00 UT. The magnetic field data obtained from the MGF instrument and plasma moment data obtained from the LEP are employed for analysis. The magnetic reconnection event is mainly identified by a large plasma flow $\left(V_{x}\right.$, Figures $1 \mathrm{~b}$ and $\left.1 \mathrm{e}\right)$ reversal from negative to positive at approximately 10:41 UT together with a reversal of the $B_{z}$ magnetic field component (Figures $1 \mathrm{a}$ and 1d). Moreover, a strong shrinking of $B_{z}$ is observed at the same time of the maximum amplitude of $V_{x}$.

[11] In the bottom part of Figure 1, data are zoomed-in within the time interval of 10:20-11:00 UT, during which the plasma flow reversal is clearly seen. Figures $1 \mathrm{c}-1 \mathrm{~d}$ plot $\mathrm{X}$ - and Z-components of the magnetic field $\left(B_{x}\right.$ and $\left.B_{z}\right)$ in the geomagnetic solar magnetosphere (GSM) coordinates. Figure 1e shows the X-component of the plasma flow $\left(V_{x}\right)$ in solid line and the parallel component of the plasma flow $\left(V_{/ /}\right)$in dashed line. The vertical arrow clearly shows the plasma flow reversal time, at approximately 10:41:40 UT. As seen from the $V_{/ /}$, the plasma flow is mainly parallel to the ambient field on the tailward side ("R4" subregion, tailward-southern hemisphere) while it is mainly antiparallel to the ambient field on the earthward side ("R3" subregion, earthward-southern hemisphere). Herein, "R1," "R2," "R3," and "R4" are the four subregions of the diffusion region in the $\mathrm{X}-\mathrm{Z}$ plane for the 2-D reconnection model as illustrated in Figure 2. The red dashed curve denotes the 0.3 Alfvenic velocity $\left(0.3 V_{a}, V_{a}=B_{0} / \sqrt{\mu_{0} \rho}\right)$. Figure $1 f$ plots the ratio of bulk velocity to the Alfvenic velocity. It will be addressed in the next context. The total plasma $\beta\left(\beta=2 \mu_{0} \cdot n k T / B_{0}^{2}\right)$ is in the range $(0.1,1)$ (Figure $1 \mathrm{~g})$ during the plasma flow reversal, suggesting that the magnetic reconnection is encountered 


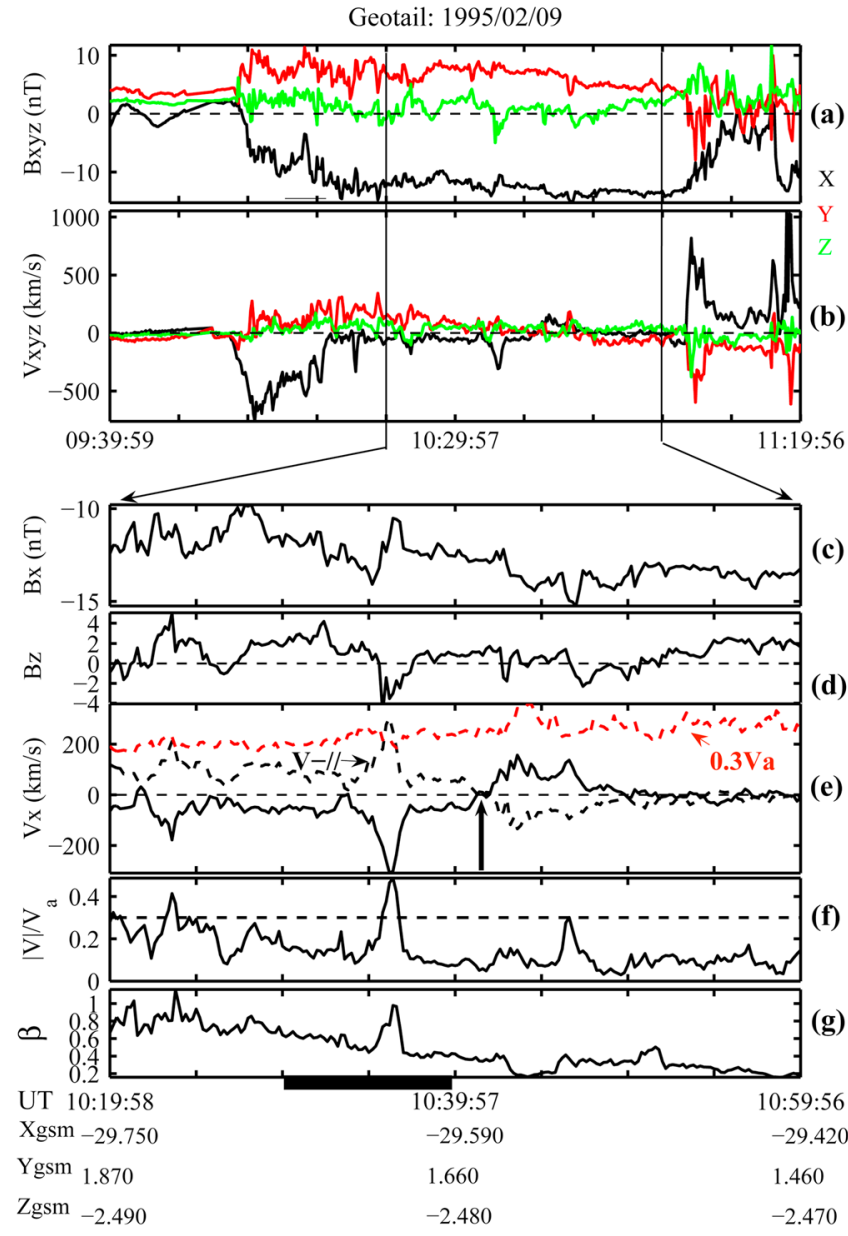

Figure 1. Overview of the magnetic reconnection observation in the near-Earth magnetotail on 9 February 1995. Figures $1 \mathrm{a}-1 \mathrm{~b}$ and $1 \mathrm{c}-1 \mathrm{f}$ represent, respectively, data within 09:40-11:20 UT (global view) and 10:20-11:00 UT (enlarged view) between the two vertical solid lines in the top part. (a-b) The magnetic field and plasma bulk velocity in the GSM coordinates. Black, red, and green lines represent X-, Y-, and Z-component, respectively. (c-d) X-component $\left(B_{x}\right)$ and Z-component of the magnetic field $\left(B_{z}\right)$; (e) X-component of the plasma flow velocity $\left(V_{x}\right)$ in solid line, and the parallel component of the plasma flow $\left(V_{/ /}\right)$in dashed line. The dashed curve in red is the 0.3 Alfvenic velocitiy. (f) Ratio of the bulk velocity to the Alfvenic velocity. The dashed horizontal line denotes the ratio of 0.3 . (g) Total plasma $\beta$.

by Geotail. The black bar in the bottom of Figure 1 marks the most interesting time interval in this study.

[12] However, since the $B_{x}$ is a little large (10-13 nT) in this interval, it is quite likely that the X-line of magnetic reconnection passed above the Geotail sometime between $\sim 10: 20$ UT and 10:50 UT. Thus, the Geotail just detected the magnetic reconnection on skimming it at the boundary layer, which is the separatrix region. It suggests that Geotail has crossed the magnetic separatrix region of this ongoing magnetic reconnection. Figure 2 schematically illustrates the trajectory of Geotail when it encounters the ongoing magnetic reconnection. The thick-solid curves with arrows in Figure 2 represent the magnetic field lines; the dashed arrowed curve stands for the Geotail trajectory with moving direction; the thin solid lines between the magnetic field lines represent the separatrix of the reconnection diffusion region. "R1," "R2," "R3," and "R4" are the four subregions of the diffusion region in the $\mathrm{X}-\mathrm{Z}$ plane of the 2-D reconnection model. The two grey balls denote the locations of Geotail relative to the magnetic reconnection diffusion region when WFC operates during the $8.75 \mathrm{~s}$ ' observation period every $275 \mathrm{~s}$. The corresponding time scopes are denoted as "W1" and "W2." Our analysis in the present study is based on the observation of WFC during these two time scopes.

[13] In this event, Geotail encounters a developing MRDR and crosses it from the tailward-southern hemisphere into the earthward-southern hemisphere on the southern-lobe-side of the diffusion region which is probably the inflow region. The flow jet at approximately 10:35 UT is of interest. The ratio of plasma flow velocity to the local Alfvenic velocity is shown in Figure 1f. As is seen, the plasma flow bursts largely and surpasses $0.3 \mathrm{Va}$ while the magnetic field decreases during the time interval of 10:35-10:37 UT. This is a sub-Alfvénic jet in the tailward outflow region near the separatrix. Due to the flapping motion of the current sheet in the reconnection region [Runov et al., 2003; Sergeev et al., 2003] or other possible reasons, the separatrix is crossed twice by Geotail.

\section{Observation and Analysis of ESWs Associated With the Separatrix}

[14] In the present event, two time periods of observations have been investigated while Geotail crosses the separatrix near the magnetic reconnection X-line. One observation period is $8.75 \mathrm{~s}$ from 10:31:09.455 UT; the other is at $8.75 \mathrm{~s}$ from 10:35:53.476 UT. According to the analysis above,

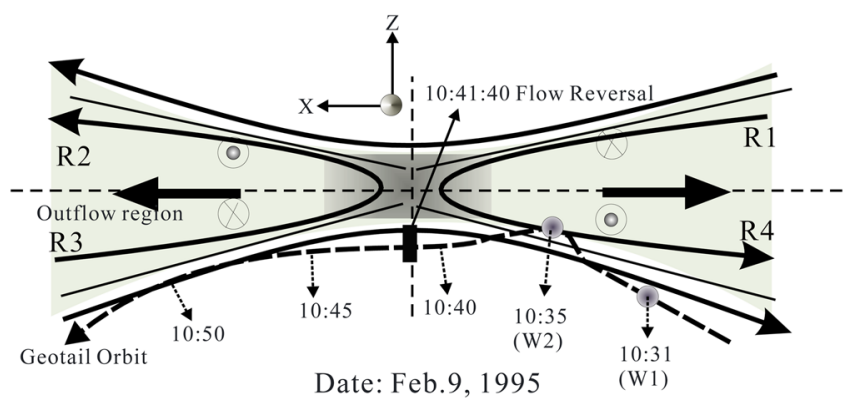

Figure 2. Illustration for the relative location of Geotail to the reconnection diffusion region and its trajectory from 10:30 UT to 11:00 UT. The reconnection diffusion region is viewed as a traditional ideal 2-D reconnection model where the $\mathrm{X}$-line is the core region of the magnetic reconnection. "R1," "R2," "R3," and "R4" are the four subregions of the diffusion region in the $\mathrm{X}-\mathrm{Z}$ plane for the 2-D reconnection model. The local magnetic field $\left(B_{y}\right)$ orientation within each region is indicated by the four balls with dot (out-of-plane) or cross (in-of-plane) in the center. The thick black arrows show the plasma outflow jet; the thick solid arrowed curves represent the magnetic field lines; the dashed allowed curve stands for the Geotail trajectory with moving direction; the thin solid lines between the magnetic field lines represent the separatrix of the reconnection diffusion region. The two black balls (W1 and W2) indicate the Geotail locations when WFC operates during the 8.75 s' duration. 


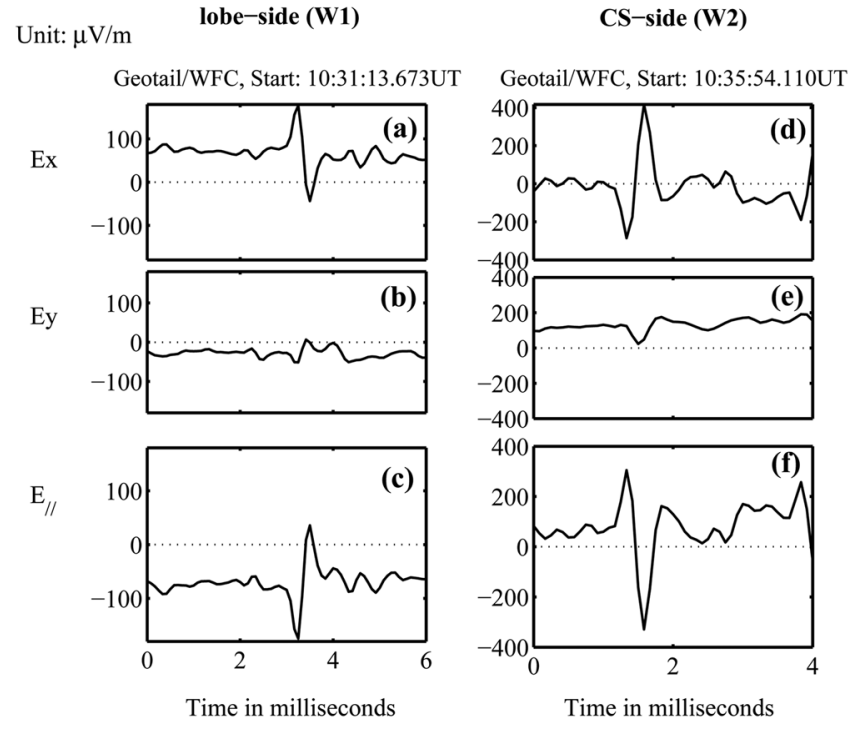

Figure 3. Enlarged view of ESWs observed during (left column) W1 and (right column) W2 events when Geotail is, respectively, located at the lobe-side and the CS-side of the separatrix. The observation time for the $\mathrm{W} 1$ case is at $10: 31: 13.673$ UT, while it is at 10:35:54.110 UT for the $\mathrm{W} 2$ case. The ambient magnetic fields in $\mathrm{W} 1$ and $\mathrm{W} 2$ ranges are $B_{0, \text { gse }}=(-11.8,8.08,1.26) \mathrm{nT}$ and $B_{0, g s e}=(-12.3,7.3$, $-2.38) \mathrm{nT}$.

the observations are made, respectively, on the lobe-side and the current sheet-side (CS-side) of the separatrix and are noted as "W1" and "W2." The orbit of Geotail crossing the magnetic separatrix adjacent to the reconnection X-line was shown in Figure 2 and will be illustrated in Figure 5. This data set is quite appropriate to study the wave activity further, especially the ESWs associated with the separatrix adjacent to the magnetic reconnection X-line.

[15] Figure 3 shows two typical cases of ESWs observed on the lobe-side and the CS-side of the separatrix (enlarged view of $\mathrm{W} 1$ and $\mathrm{W} 2)$. The panels in left $(\mathrm{a}-\mathrm{c})$ and right (d-f) are, respectively, for W1 and W2 observation. Panels (a-b) show the electric field components $E_{x}$ and $E_{y}$ (versus time) yielded from $E_{x}=E_{u} \cdot \sin (\theta)+E_{v} \cdot \cos (\theta)$ and $E_{y}=-E_{u} \cdot \cos (\theta)+E_{v} \cdot \sin (\theta)$, where $E_{u}$ and $E_{v}$ (not shown) are the electric components directly measured by the two antennas onboard Geotail. The $E_{U}$ and $E_{V}$ are in satellite-based coordinates (hereafter called sc-coordinates), which is very similar to the GSE coordinates during the mission of Geotail. The variable $\theta(t)$ is the spin angle between the $\mathrm{U}$ (or $\mathrm{V}$ ) axis in one time $\left(t=t_{1}\right)$ to the $\mathrm{U}$ (or $\mathrm{V}$ ) axis of the original status $\left(t=t_{0}\right)$ within the spin plane of Geotail. In this case, the plot of $E_{x}(t)$ and $E_{y}(t)$ can be viewed as data in the Geomagnetic Solar Ecliptic
(GSE) coordinate set. $E_{z}$ is usually set to be zero since the ambient field is mainly parallel to the $x-y$ plane in this analysis. The bipolar structure of ESWs is identified by both the original data $\left(E_{U}\right.$ or $\left.E_{V}\right)$ and the transformed data $\left(E_{x}\right.$ or $\left.E_{y}\right)$. Commonly, the bipolar structure is mainly identified by $E_{x}$ or $E_{y}$. Figure $3 \mathrm{c}$ shows the parallel component of the electric field $\left(E_{/ /}\right)$which is obtained from $E_{/ /}=E \cdot B / B t$.

[16] The observation of ESWs associated with the separatrix has been reported [e.g., Kojima et al., 1999b; Cattell et al., 2005]; however, we will focus herein on polarizations of the ESWs on both sides of the separatrix. The bipolar solitary structure of the parallel component of the ESW for the W1 observation exhibits a first-negative-then-positive variation bipolar solitary structure, while for the W2 observation case, it has an opposite variation. For convenience, we name the first-negative-then-positive waveform as "type-A-ESW," and the opposite one as "type-B-ESW."

[17] Many cases of ESWs similar to those of Figure 3 have been identified and analyzed within the W1 and W2 time intervals. Statistical results are listed in Table 1. In summary, about $85 \%$ (40/47) of the ESWs in W1 period are with the same polarization of type-A ESWs; while in W2 period, the percentage of type-B-ESWs can reach $83 \%$ (49/59). Figure 4 shows the waveform $E_{x}-E_{y}$ during the W2 period when Geotail is located at the CS-side of the separatrix. Figure 4a plots $E_{x}$ and $E_{y}$ versus time, respectively, in a time span of $300 \mathrm{~ms}$ starting from 10:30:53.955 UT. Tens of spiky solitary structures with small pulse width can be differentiated from $E_{x}(t)$ and $E_{y}(t)$ profiles in Figures $4 \mathrm{a}$ and $4 \mathrm{~b}$. An enlarged view is plotted in Figure 4c. Almost all ESWs within the W2 interval of $300 \mathrm{~ms}$ have the same type-B polarization.

[18] The generation source of ESWs in the magnetosphere is an important issue and has been investigated by many authors [e.g., Pickett et al., 2005; Cattell et al., 2005]. However, as for the generation of the ESW associated with magnetic reconnection, only a few evidences have been presented based on satellite observation [e.g., Farrell et al., 2002; Cattell et al., 2005] apart from simulation [Drake et al., 2003; Lapenta et al., 2011].

[19] In the present event, the opposite polarization of ESWs on the lobe-side and the CS-side of the separatrix near the reconnection X-line presents a new way to analyze the source regions of ESWs in the MRDR and their propagation directions. As mentioned above, ESWs on the lobe-side of the separatrix are mainly of type-A, while on the CS-side they are of type-B. The different polarizations between these two kinds suggest that the propagating directions of the solitary potential along the ambient field are opposite. As is studied by Kojima et al. [1999a] and indicated by Shin et al. [2008], the propagation direction of the ESWs can be evaluated from the phase of the bipolar ESW waveforms

Table 1. Statistical Results of ESWs Observed in the Lobe-Side (W1) and Current Sheet-Side (W2) of Magnetic Separatrix, Respectively

\begin{tabular}{lccccccc}
\hline Time & Num & Type-A & Type-B & $\begin{array}{c}\text { Amplitude (Mean) } \\
(\mu \mathrm{V} / \mathrm{m})\end{array}$ & $\begin{array}{c}\text { Amplitude (Max) } \\
(\mu \mathrm{V} / \mathrm{m})\end{array}$ & $\begin{array}{c}\text { Pulse-width (Mean) } \\
(\mathrm{ms})\end{array}$ & $\begin{array}{c}\text { Pulse Width } \\
(\mathrm{Max})(\mathrm{ms})\end{array}$ \\
\hline W1 & 47 & $40(85.1 \%)$ & $7(14.9 \%)$ & 86.6 & 278.3 & 1.03 & 2.48 \\
W2 & 59 & $10(17 \%)$ & $49(83 \%)$ & 501.8 & 1440.1 & 1.25 & 6.25 \\
\hline
\end{tabular}


LI ET AL.: ESWs ANALYSIS IN SEPARATRIX

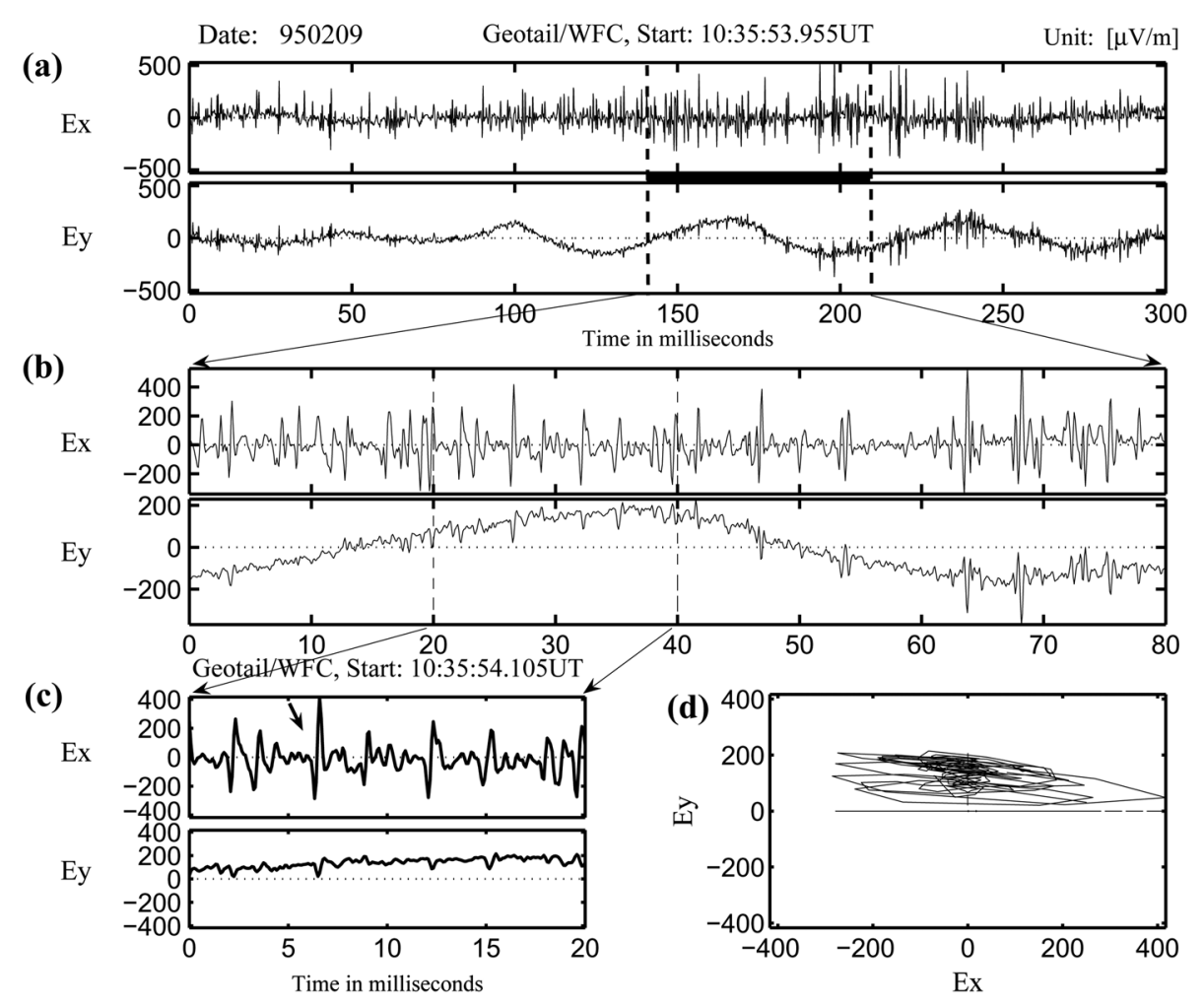

Figure 4. (a) $300 \mathrm{~ms}$ data of $E_{x}$ and $E_{y}$ fields observed during the W2 event when Geotail is located at the CS-side of the separatrix. (b) Zoom-in showing the waveform within the time span marked by the two vertical lines defined in Figure 4a. (c) $20 \mathrm{~ms}$ data observed from 10:35:54.105 UT which is the expanded scale of waveform between the two vertical dashed lines defined in Figure $4 \mathrm{~b}$. For reference, the arrow in Figure 4c marks the bipolar waveform which has been analyzed in (right column) Figure 3. Figure 4d shows the polarity $\left(E_{x}-E_{y}\right)$ of the waveform.

under the assumption of the polarity of ESW potentials. When we assume the positive potentials for the ESWs, the propagation direction of type-A ESWs and type-B ESWs are, respectively, antiparallel and parallel to the ambient magnetic field (illustrated in Figure 5a). In summary, the information on the propagating directions relative to the ambient field can be obtained from the polarizations of ESWs, i.e., the type-A-ESW refers to that "antiparallel to $\mathrm{B}_{0}$," and the type-B-ESW refers to that "parallel to $\mathrm{B}_{0}$." In this case, the separatrix is on the tailward-southern side of the X-line, thus the ambient field is tailward. The ESWs observed on the lobe-side are antiparallel to the ambient field (earthward, i.e., toward the reconnection X-line) while those observed on the CS-side are parallel (tailward, i.e., outward and away from the reconnection X-line).

[20] Figure $5 \mathrm{~b}$ illustrates the tailward side of the magnetic reconnection $\mathrm{X}$-line for the present analysis. On the basis of ESWs observations on both sides of the separatrix near the reconnection X-line, it also illustrates the polarizations of ESWs in the ideal 2-D magnetic reconnection model in $X-Z$ plane in the near-earth magnetotail. The thick (thin) arrowed lines stand for magnetic field lines on the lobe-side (CS-side) of the separatrix, while the grey bar between these two kinds of lines represents the separatrix. The W1 and W2 observation are, respectively, operated when Geotail is located on the lobe-side and the CS-side of the separatrix. The waveforms in the bipolar structure represent the ESWs observed by the Geotail.
[21] The opposite polarizations of ESWs in the lobe-side and the CS-side of the separatrix adjacent X-line can be used as a "tracer" to derive the source region of ESWs associated with tail reconnection. However, the observation here only confirms the case in the tailside-southern region of the X-line (waveform in blue). So, a future work will be necessary to extend the present analysis to other regions of the MRDR.

[22] In order to study more carefully the ESWs associated with the separatrix, a simple statistics is performed and is summarized in Table 1. Columns 2 to 4 list, respectively, the total number of type-A-ESW and type-B-ESW. The pulse widths for ESWs observed on the lobe-side and the CS-side of the separatrix are listed in columns 7 and 8 of Table 1. The maximum pulse width of ESWs in the CS-side is $6.25 \mathrm{~ms}$, while it is $2.48 \mathrm{~ms}$ for the ESWs in the lobe-side. The pulse width of the bipolar solitary structure observed near the X-line of diffusion region is consistent with those observed mainly in the PSBL in the vicinity of the diffusion region which is in the scope of 1-5 ms [Li et al., 2009].

[23] A large difference is seen from the amplitudes of ESWs observed on the lobe-side and the CS-side of the separatrix. Columns 5 and 6 list the maximum and mean values of the ESWs' amplitudes observed in W1 (lobe-side) and W2 (CS-side). On the lobe-side of the separatrix, the average amplitude is just $86.6 \mu \mathrm{V} / \mathrm{m}$, while the value on the CS-side is $501.8 \mu \mathrm{V} / \mathrm{m}$, i.e., over 6 times longer than that on the lobe-side. The maximum amplitude is $1440.1 \mu \mathrm{V} / \mathrm{m}$ for ESWs on the CS-side and is again over 6 times longer than 
(a)

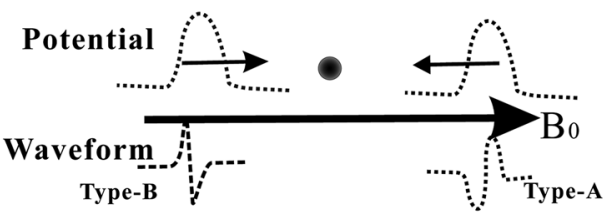

(b)

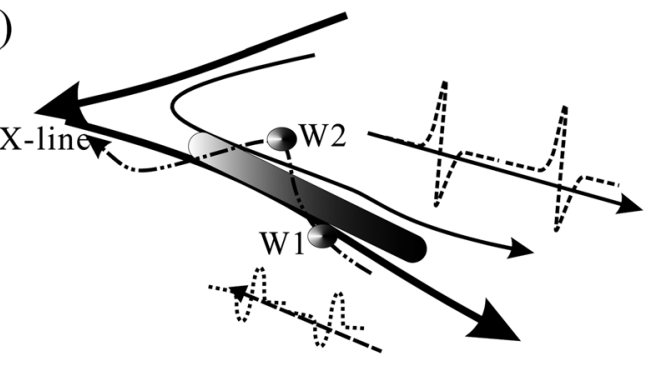

Figure 5. (a): Sketch of the propagation of ESWs along the ambient field according to their waveform (type-A or typeB); (b) Illustration of the polarization of ESWs in the ideal 2-D magnetic reconnection model on the $\mathrm{X}-\mathrm{Z}$ plane in the near-earth magnetotail. The thick (thin) lines with arrows stand for the magnetic field lines on the lobe-side (CS-side) of the plasma sheet, while the grey area represents the separatrix transition layer. The dashed arrow line marks the trajectory of Geotail. The waveforms of bipolar structures represent the different ESWs patterns; the thin black arrows represent their propagation direction, respectively.

that on the lobe-side. The ESWs' amplitude is likely to reflect the energy of the driving electrons and the vechanism that limits the wave growth (issues that are discussed in many simulation and theory papers). The large difference between amplitudes of ESWs observed on the lobe-side and the current sheet-side of the separatrix suggests that the electrostatic energy and the kinetic energy of electrons forming the electron holes moving outward from reconnection X-line are much larger than those moving inward.

\section{Analysis of Particle Data}

[24] Figure 6 shows the time histories of the electron differential flux from seven different channels of LEP-EA-e by Geotail. The color bar is unified for the sake of making comparison between different channels. Top to bottom panels show the data collected from the channels in the detector LEP-EA corresponding to the incident elevation angles of $0^{\circ}, \pm 22.5^{\circ}, \pm 45^{\circ}$, and $\pm 67.5^{\circ}$. Channel-1 (CH.1) denotes the detector looking toward the north-most elevation angle $(+67.5)$ and hence measuring the southward-going particles. Channel-4 (CH.4) is the center detector in the equator plane (roughly ecliptic plane). Channel-7 (CH.7) is the detector opposite to Channel-1 and measures the northward-going particles [Mukai et al., 1994]. The measured data have been corrected by the g-factor of each channel for EA-e (refer to Table 2 in Mukai et al. [1994]). Moreover, we use the efficiency of $\mathrm{CH} .1$ to $\mathrm{CH} .7$ to fully correct the count data, dividing the g-factor corrected count by efficiency of $\mathrm{CH} .1$ to CH.7, which are $0.49,0.40,0.35,0.34,0.30,0.39$, and 0.48 , respectively [Mukai et al., 1994]. Note that the low energy portion in the electron counts from the lowest five energy steps (i.e., Step 31: 43.4-76.6 eV; Step 30: 76.6-134.3 eV; Step 29: 134.3-213.0 eV; Step 28: 213.0-281 eV; Step 27: $281-349.1 \mathrm{eV}$ ) have been filtered and are not shown since it is too great and will enshroud the electrons in higher energy flux passes.

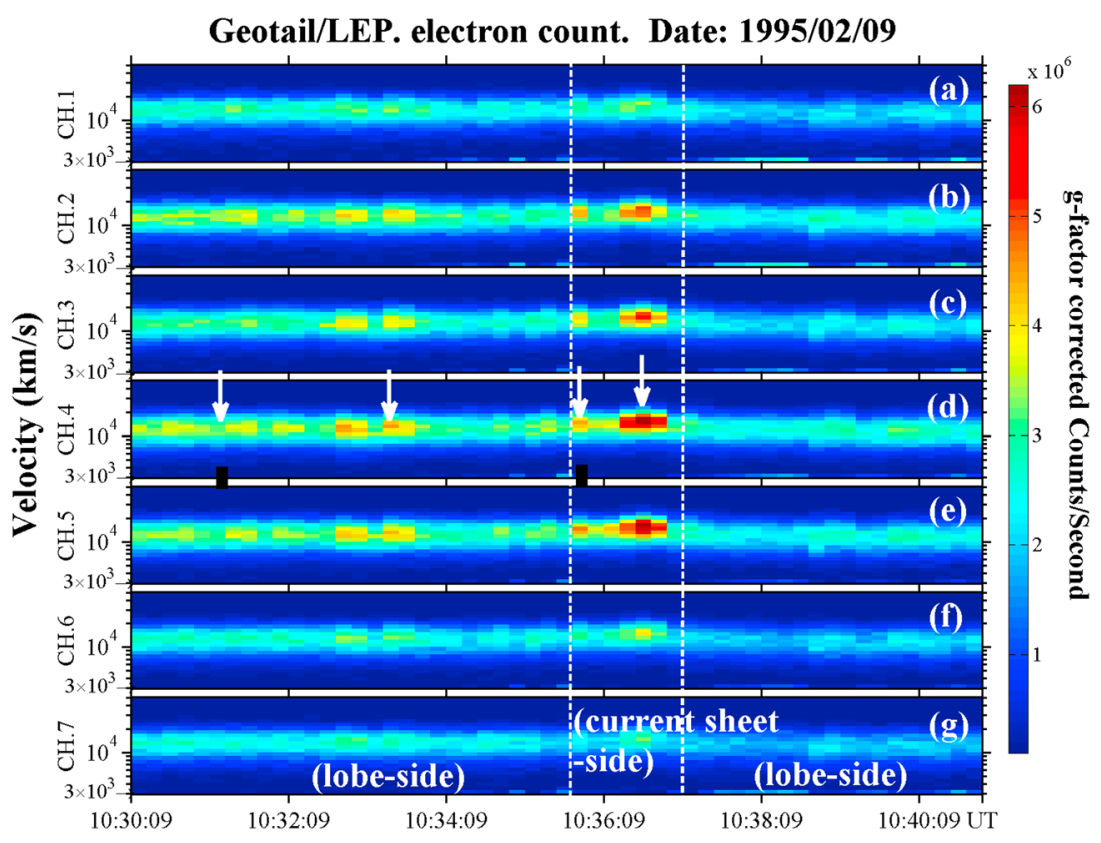

Figure 6. Time histories of the electron differential flux (g-factor corrected) from the seven different channels of LEP-EA-e by Geotail on 9 February 1995. Y axis in each panel is the velocity channel, from 2959 to 51,205 (km/s), which stands for energy bins [Mukai et al., 1994]. For each channel, the electron account is integrated in all azimuthal directions. Refer to text for detail. 

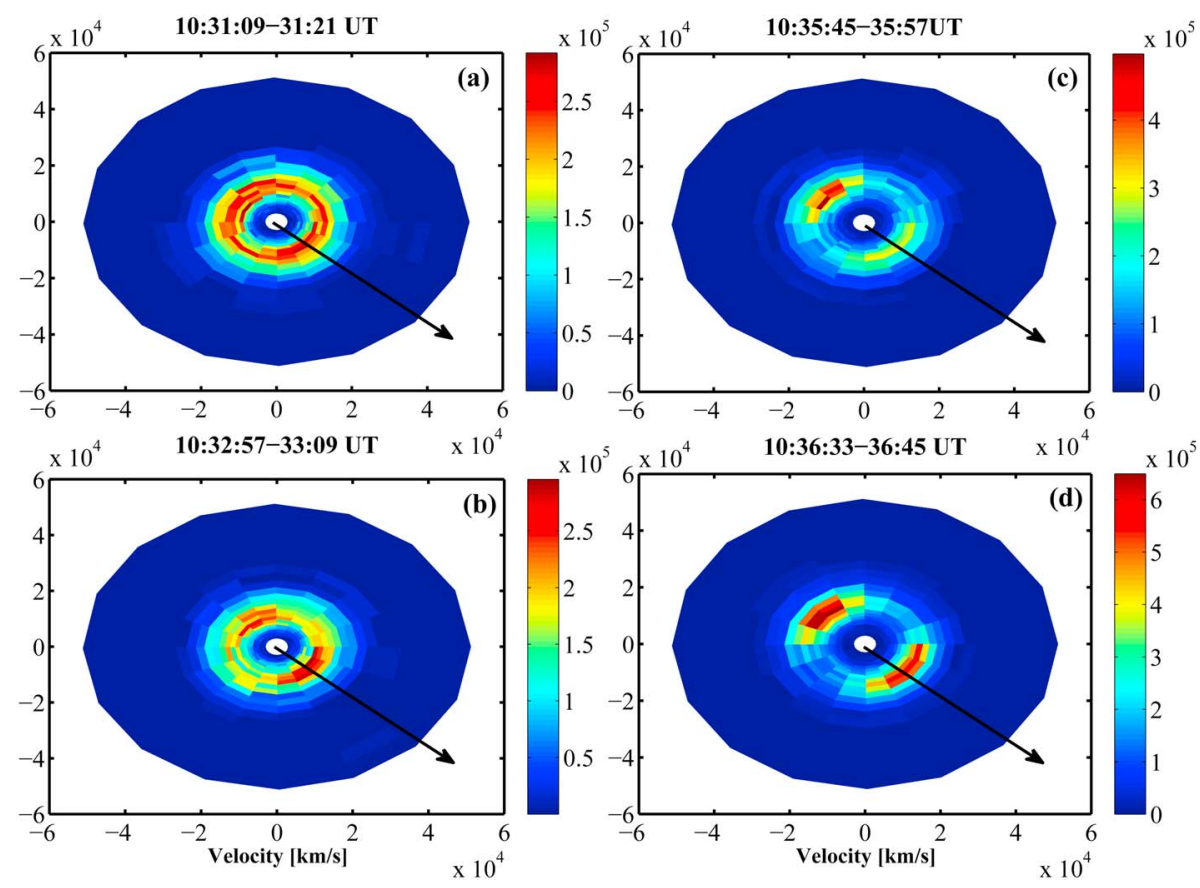

Figure 7. Two-dimensional cut in Channel 4 of 3-D distribution of electron differential flux (g-factor corrected) as observed by LEP instrument. The unit of the colorbar is the same as that in Figure 6 . The subplots are denoted by the four white arrows in Figure 6. Figures $7 \mathrm{a}$ and $7 \mathrm{~b}$ are for lobe-side while Figures 7c and 7d are for current sheet-side. The WFC observation time spans "W1" and "W2" correspond to the plots (a) and (b). The black arrows in the subplots indicate the direction of ambient magnetic field. Refer to text for detail.

[25] The two vertical white dashed lines in Figure 6 separate the observation time span into three stages, corresponding to the regions relative to the magnetic separatrix as lobe-side, CS-side, and then the lobe-side again. It shows clearly that the electron flux with velocity around $10,000 \mathrm{~km} / \mathrm{s}$ enhances mainly in channel 4 and 5 (CH.4-5, Figure 6d-6e), which is roughly in the ecliptic plane, i.e., in the plane parallel to the ambient magnetic field. Moreover, it can be seen in Figure 6d that the electron flux enhances largely on the CS-side of the separatrix while it is only slightly enhanced on the lobe-side. The maximum of electron flux on the CS-side of the separatrix is $\sim 6.5 \times 10^{6}$ on $10: 36: 33$ UT while on the lobe-side it is $\sim 4.5 \times 10^{6}$ on $10: 32: 45$ UT.

[26] To investigate whether electron beam exists and their direction, we have surveyed the two-dimensional cut distribution of electron in the plane parallel to the ambient magnetic field, i.e., in Channel-4 for all the time interval as shown in Figure 6d. Figures $7 \mathrm{a}-7 \mathrm{~d}$ show the twodimensional cut distribution, respectively, for four integrated time spans as indicated by the four downward white arrows in Figure 6. Figures $7 \mathrm{a}-7 \mathrm{~b}$ are the electron distributions for the observation on the lobe-side of the separatrix, while the other two panels are those on the CS-side. The distribution in each panel is obtained in one LEP integrated period of $12 \mathrm{~s}$. The electron flux is divided into 16 levels of energy and 16 azimuthal angles scales; however, as addressed in Figure 6, the lowest five energy levels have been filtered. Note that Figure $7 \mathrm{a}$ is plotted in the time interval including the "W1" observation and Figure 7c corresponding to "W2" observation. For Figures $7 \mathrm{~b}, 7 \mathrm{c}$, and $7 \mathrm{~d}$, the electron distribution function is strongly elongated along the ambient magnetic field (the black arrows) at higher energy levels with velocities $\sim 10,000 \mathrm{~km} / \mathrm{s}$, suggesting the existence of bidirectional or unidirectional electron beams, which is consistent with the Buneman instability [Matsumoto et al., 2005].

[27] The electron beam in the separatrix has been widely studied by simulations and observations. A beam of lowenergy electrons flowing toward the reconnection region near the separatrix was previously identified in observations [Fujimoto et al., 2001], as well as in kinetic simulations [Pritchett, 2001; Hoshino et al., 2001]. Deng et al. [2004] have reported direction reversal of electron beam in the PSBL near the MRDR, which is an evidence of the Hall current loop in the magnetic reconnection diffusion region. They have indicated that the electron beams flow into the reconnection X-line from the lobe-side of the separatrix. Nagai et al. [2011] also indicated that the electrons show basically bidirectional field-aligned distributions near the separatrix layer. In this case as shown in Figures $7 b, 7 c$, and $7 \mathrm{~d}$, the Geotail/LEP data show basically bidirectional field-aligned distributions or unidirectional field-aligned distributions in the region near the separatrix layer of vmagnetic reconnection. This observation of electron beam associated with the separatrix is consistent with those observed by Cluster [Cattell et al., 2005] and Geotail [Nagai et al., 2011].

[28] The ESWs are commonly regarded to be generated by the electron two-stream instabilities and/or bump-on-tail instability [Omura et al., 1994, 1996, 1999]. Moreover, by examining the electron distributions on all the satellites of Cluster, Cattell et al. [2005] suggested that the electron holes 
are excited in the separatrix region, near the outer edge of the current sheet, where there are narrow electron beams, either unidirectional or counterstreaming. Fujimoto and Machida [2006] also suggested that the electron two-stream instability in association with magnetic reconnection is also responsible for the generation of the electrostatic solitary waves that have been frequently detected in the boundary region. The observation of ESWs and unidirectional electron beam is similar to the previous result.

[29] In summary, our analysis on the LEP data shows that, electron beam associated with ESWs parallel to the ambient field on the CS-side of separatrix is strong while it is weak on the lobe-side region. Furthermore, the direction of the electron beam on the lobe-side of the separatrix is mainly antiparallel to the ambient magnetic field (Figure 7b) and it is mainly parallel on the CS-side (Figure 7c). Both are consistent with the propagation of ESWs which is in agreement with the generation mechanism of ESWs which is suggested to be related to electron beams.

\section{Discussion and Concluding Remarks}

[30] In the present work, a sub-Alfve'nic jet in the tailward outflow region near the separatrix of the magnetic reconnection is observed by Geotail on 9 February 1995. Several tens of electrostatic solitary waves/pulses (ESWs) are observed, respectively, on the lobe-side and the CS-side of the separatrix. The detailed analysis on the polarizations of ESWs associated with the separatrix has been performed. The results are summarized as follows:

[31] 1. The ESWs on the CS-side are of type-B with direction outward (toward to the tailward) while on the lobe-side they are of type-A directed to X-line. The amplitude of ESWs on the CS-side is about 6 times more than those on the lobe-side, suggesting that energies flowing outward from the reconnection X-line are much larger than those flowing inward. This feature can be helpful for understanding the role of ESWs in the process of fast energy release associated to the magnetic reconnection.

[32] 2. The analysis on the LEP data shows that electron beams associated with ESWs, which are parallel to the ambient magnetic field, are much stronger on the CS-side than on the lobe-side of the separatrix. Furthermore, the direction of the electron beam on the lobe-side of the separatrix is mainly antiparallel to the ambient magnetic field and it is mainly parallel on the CS-side. Both are consistent with the propagation of ESWs which is in agreement with the generation mechanism of ESWs which is suggested to be related to electron beams.

[33] Che et al. [2010] explored the current-driven turbulence and associated electron heating in the dissipation region during $3 \mathrm{D}$ magnetic reconnection with a guide field by employing particle-in-cell simulations and kinetic theory. They found that the turbulence was dominated by the Buneman and lower hybrid instabilities in the late stage of simulation. Both produce electron holes that co-exist but have very different propagation speeds. However, in this work, the generation mechanism of the ESWs cannot be clearly distinguished. Furthermore, Wang et al. [2010] also concluded that during the crossing of the separatrix with the reversal of plasma flow and Hall magnetic fields, strong electrostatic LHD mode was observed. Our analysis has not referred to the relationship between the lower hybrid instabilities and ESWs which will be left to future work.

[34] Recent results suggest that ESWs may be generated in the reconnection diffusion region and propagate along (parallel or antiparallel to) the ambient field in the outward flow region. This feature is consistent with the simulation result of Drake et al. [2003]. The opposite polarizations of ESWs on the lobe-side and the CS-side of the magnetic separatrix near the reconnection X-line can be used as a "tracer" to derive the source region of the ESWs associated with tail reconnection. However, a future work is necessary to extend the propagation characteristics of ESWs on both sides of the separatrix in regions other than the tailsidesouthern region of the X-line presented herein.

[35] For the present cases of ESWs observed on both sides of the separatrix, plasma moment data obtained from the LEP instrument show that the temperature of ions at W1 (lobe-side of the separatrix) and W2 (CS-side of the separatrix) observation periods are: (W1) $T_{i y y}=1027 \mathrm{eV}, T_{i z z}=862 \mathrm{eV}$, (W2) $T_{i y y}=1937 \mathrm{eV}, T_{i z z}=1194 \mathrm{eV}$. Because the spin plane is mainly parallel to the $\mathrm{X}-\mathrm{Y}$ plane, $T_{i / /} \approx T_{i y y}$ and $T_{i \perp} \approx T_{i z z}$. $T_{i / /, W 2} / T_{i / /, W 1} \approx 1.9$, while $T_{i \perp, W 2} / T_{i \perp, W 1} \approx 1.4$. Since both average and maximum amplitudes of ESWs in W2 observation period (on the CS-side of the separatrix) are about 6 times larger than those in $\mathrm{W} 1$ observation period (on the lobe-side), the parallel temperature $T_{i / /}$ might be an important parameter to influence the intensity of the ESWs. However, we do not have result on this point. Simulation work might be helpful to classify the relationship between the parallel temperature and the intensity of ESWs.

[36] Moreover, there are several restrictions in the present study. First, the observations of ESWs on both sides of the separatrix adjacent to the reconnection X-line are limited because the instrument has some constraints since the WFC experiment is operational only lasting $8.75 \mathrm{~s}$ every $275 \mathrm{~s}$. Then, it is necessary to perform a more complete statistical analysis to obtain better understanding on this issue. Second, our study is based on mono-point observations. An extensive study with multipoints observations based on CLUSTER and THEMIS data [Angelopoulos, 2008] is necessary and is currently undertaken.

[37] Acknowledgments. This work is supported by the National Natural Science Foundation of China under Grant 41304132, the PostDoctoral Research Program of China (42956), the 53-class General Financial Grant from the China Postdoctoral Science Foundation under Grant of 2013M532115, and the National Basic Research Program of China. The authors thank all of the members of the Geotail team for the high quality data and successful operation. This work has been developed while both co-authors (S.Y. Li and B. Lembege) were at RISH Laboratory of Kyoto University which is thanked for its hospitality and warm welcome. S. Y. Li appreciates T. Nagai very much for help on examining the draft.

[38] Philippa Browning thanks S. Gary and two anonymous reviewers for their assistance in evaluating this paper.

\section{Reference}

André, M., A. Vaivads, S. C. Buchert, A. N. Fazakerley, and A. Lahiff (2004), Thin electron-scale layers at the magnetopause, Geophys. Res. Lett., 31, L03803, doi:10.1029/2003GL018137.

Angelopoulos, V. (2008), The THEMIS mission, Space Sci. Rev., 141, 5-34, doi:10.1007/s11214-008-9336-1.

Bale, S. D., P. J. Kellogg, D. E. Larson, R. P. Lin, K. Goetz, and R. P. Lepping (1998), Bipolar electrostatic structures in the shock transition region: Evidence of electron phase space holes, Geophys. Res. Lett., 25, 2929-2932. Bernstein, I. B., J. M. Greene, and M. D. Kruskal (1957), Exact nonlinear plasma oscillations, Phys. Rev. Lett., 108, 546-550. 
Cattell, C. A., J. Dombeck, and J. R. Wygant (1999), Comparisons of Polar satellite observations of solitary wave velocities in the plasma sheet boundary and the high altitude cusp to those in the auroral zone, Geophys. Res. Lett., 26, 425-428, doi:10.1029/1998GL900304.

Cattell, C., C. Neiman, J. Dombeck, J. Crumley, J. Wygant, C. A. Kletzing, W. K. Peterson, F. S. Mozer, and M. André (2003), Large amplitude solitary waves in and near the Earth's magnetosphere, magnetopause and bowshock: Polar and cluster observations, Nonlinear Processes Geophys., 10, $13-26$

Cattell, C., et al. (2005), Cluster observations of electron holes in association with magnetotail reconnection and comparison to simulations, J. Geophys. Res., 110, A01211, doi:10.1029/2004JA010519.

Che, H., J. F. Drake, and M. Swisdak (2010), Electron holes and heating in the reconnection dissipation region, Geophys. Res. Lett., 37, L11105, doi:10.1029/2010GL043608.

Chen, L.-J., and G. K. Parks (2002), BGK electron solitary waves in 3D magnetized plasma, Geophys. Res. Lett., 29(9), 1331, doi:10.1029/ $2001 \mathrm{GL} 013385$.

Deng, X. H., H. Matsumoto, H. Kojima, T. Mukai, R. R. Anderson, W. Baumjohann, and R. Nakamura (2004), Geotail encounter with reconnection diffusion region in the Earth's magnetotail: Evidence of multiple X lines collisionless reconnection?, J. Geophys. Res., 109, A05206, doi:10.1029/2003JA010031.

Deng, X. H., et al. (2009), Dynamics and waves near multiple magnetic nul points in reconnection diffusion region, J. Geophys. Res., 114, A07216, doi:10.1029/2008JA013197.

Drake, J. F., M. Swissdak, C. Cattell, M. A. Shay, B. N. Rogers, and A. Zeiler (2003), Formation of electron holes and particle energization during magnetic reconnection, Science, 299, 873-877.

Ergun, R., et al. (1998), FAST satellite observations of large-amplitude solitary wave structures, Geophys. Res. Lett., 25, 2041-2044.

Farrell, W. M., M. D. Desch, M. L. Kaiser, and K. Goetz (2002), The dominance of electron plasma waves near a reconnection X-line region, Geophys. Res. Lett., 29(19, 1902), doi:10.1029/2002GL014662.

Fujimoto, K., and S. Machida (2006), A generation mechanism of electrostatic waves and subsequent electron heating in the plasma sheet-lobe boundary region during magnetic reconnection, J. Geophys. Res., 111, A09216, doi:10.1029/2005JA011542.

Fujimoto, M., T. Nagai, N. Yokokawa, Y. Yamade, T. Mukai, Y. Saito, and S. Kokubun (2001), Tailward electrons at the lobe-plasma sheet interface detected upon dipolarizations, J. Geophys. Res., 106(A10), 21,255-21,262, doi:10.1029/2001JA900011.

Hoshino, M., T. Mukai, T. Terasawa, and I. Shinohara (2001), Suprathermal electron acceleration in magnetic reconnection, J. Geophys. Res., 106(A11), 25,979-25,997, doi:10.1029/2001JA900052.

Khotyaintsev, Y., V. Vaivads, A. Retinó, A. André, C. J. Owen, and H. Nilsson (2006), Formation of inner structure of a reconnection separatrix region, Phys. Rev. Lett., 97, 205003, doi:10.1103/PhysRevLett.97.205003.

Khotyaintsev, Y. V., A. Vaivads, M. André, M. Fujimoto, A. Retinò, and C. J. Owen (2010), Observations of slow electron holes at a magnetic reconnection site, Phys. Rev. Lett., 105, 165002, doi:10.1103/PhysRevLett.105.165002.

Kojima, H., et al. (1994), Relation between electrostatic solitary waves and hot plasma flow in the plasma sheet boundary layer: GEOTAIL observations, Geophys. Res. Lett., 21, 2919-2922.

Kojima, H., H. Matsumoto, S. Chikuba, S. Horiyama, M. Ashour-Abdalla, and R. R. Anderson (1997), GEOTAIL waveform observations of broadband/narrowband electrostatic noise in the distant tail, J. Geophys. Res., 102, $14,439-14,455$.

Kojima, H., Y. Omura, H. Matsumoto, K. Miyaguti, and T. Mukai (1999a), Characteristics of electrostatic solitary waves observed in the plasma sheet boundary: Statistical analysis, Nonlinear Process Geophys., 6, 179-186.

Kojima, H., K. Ohtsuka, H. Matsumoto, Y. Omura, R. R. Anderson, Y. Saito, T. Mukai, S. Kokubun, and T. Yamamoto (1999b), Plasma waves in slow-mode shocks observed by GEOTAIL spacecraft, Adv. Space Res., $24(1), 51-54$.

Kokubun, S., T. Yamamoto, M. H. Acuna, K. Hayashi, K. Shiokawa, and H. Kawano (1994), The GEOTAIL magnetic field experiment, J. Geomag. Geoelectr., 46, 7-21.

Lapenta, G., S. Markidis, A. Divin, M. V. Goldman, and D. L. Newman (2011), Bipolar electric field signatures of reconnection separatrices for a hydrogen plasma at realistic guide fields, Geophys. Res. Lett., 38, L17104, doi:10.1029/2011GL048572.

Li, S. Y., X. H. Deng, M. Zhou, R. X. Tang, K. Liu, H. Kojima, and H. Matsumoto (2009), Statistical study of electrostatic solitary waves associated with reconnection: Geotail observations, J. Adv. Space Res., 43(3), 394-400, doi:10.1016/j.asr.2008.05.013.

Li, S. Y., X. H. Deng, M. Zhou, Z.-G. Yuan, J.-F. Wang, X. Lin, M.-H. Lin, and S. Fu (2010), Cluster observation of Electrostatic Solitary Waves around magnetic null point in thin current sheet, Chin. Phys. Lett., 27(1), 019,401 .
Li, S. Y., S. F. Zhang, X. H. Deng, and H. Cai (2012), Spatial evolution of Electrostatic Solitary Waves along plasma sheet boundary layer adjacent to magnetic reconnection X-line, Chin. Phys. Lett., 29(8), 089,402.

Li, S. Y., S. F. Zhang, X. H. Deng, and H. Cai (2013a), Large bi-polar signature in a perpendicular electric field of two-dimensional electrostatic solitary waves associated with magnetic reconnection: Statistics and discussion, Chin. Phys. Lett., 30(1), 019,401.

Li, S. Y., S. F. Zhang, H. Cai, X. Q. Chen, and X. H. Deng (2013b), Statistics of the tri-polar electrostatic solitary waves within magnetic reconnection diffusion region in the near-Earth magnetotail, J. Geophys. Res. Space Physics, 118, 2911-2918, doi:10.1002/jgra.50209.

Matsumoto, H., H. Kojima, T. Miyatake, Y. Omura, M. Okada, I. Nagano, and M. Tsutsui (1994a), Electrostatic solitary waves ESW in the magnetotail: BEN waveforms observed by Geotail, Geophys. Res. Lett., 21, 2915-2918.

Matsumoto, H., I. Nagano, R. R. Anderson, H. Kojima, K. Hashimoto, M. Tsutsui, T. Okada, I. Kimura, Y. Omura, and M. Okada (1994b), Plasma wave observations with Geotail Spacecraft, J. Geomagn. Geoeletr., 46, 59-95.

Matsumoto, H., L. A. Frank, Y. Omura, H. Kojima, W. R. Paterson, M. Tsutsui, R. R. Anderson, S. Horiyama, S. Kokubun, and T. Yamamoto (1999), Generation mechanism of ESW based on GEOTEIL plasma wave observation, plasma observation and particle simulation, Geophys. Res. Lett., 26(3), 421-424.

Matsumoto, H., X. H. Deng, H. Kojima, and R. R. Anderson (2003), Observation of Electrostatic Solitary Waves associated with reconnection on the dayside magnetopause boundary, Geophys.Res.Lett., 30(6), 1326, doi:10.1029/2002GL016319.

Matsumoto, H., M. Iwata, and T. Sugiyama (2005), Generation mechanism of electrostatic waves in the upstream and shock transition regions of quasi-parallel shocks, J. Geophys. Res., 110, A01104, doi:10.1029/ 2004JA010481.

Mozer, F. S., R. Ergun, M. Temerin, C. Cattell, J. Dombeck, and J. Wygant (1997), New features in time domain electric field structures in the auroral acceleration region, Phys. Rev. Lett., 79(7), 1281-1284.

Mukai, T., S. Machida, Y. Saito, M. Hirahara, T. Terasawa, N. Kaya, T. Obara, M. Ejiri, and A. Nishida (1994), The low energy particle (LEP) experiment onboard the Geotail satellite, J. Geomag. Geoelectr., 46, 669-692

Nagai, T., I. Shinohara, M. Fujimoto, A. Matsuoka, Y. Saito, and T. Mukai (2011), Construction of magnetic reconnection in the near-Earth magnetotail with Geotail, J. Geophys. Res., 116, A04222, doi:10.1029 2010JA016283.

Omura, Y., H. Kojima, and H. Matsumoto (1994), Computer simulation of Electrostatic Solitary Waves: A nonlinear model of broadband electrostatic noise, Geophys. Res. Lett., 21(25), 923-2926.

Omura, Y., H. Matsumoto, T. Miyake, and H. Kojima (1996), Electron beam instabilities as generation mechanism of electrostatic solitary waves in the magnetotail, J. Geophys. Res., 101, 2685-2697.

Omura, Y., H. Kojima, N. Miki, T. Mukai, H. Matsumoto, and R. Anderson (1999), Electrostatic Solitary Waves carried by diffused electron beams observed by GEOTAIL spacecraft, J. Geophys. Res., 104(A7), $14,627-14,637$.

Pickett, J. S., J. D. Menietti, D. A. Gurnett, B. Tsurutani, P. M. Kintner, E. Klatt, and A. Balogh (2003), Solitary potential structures observed in the magnetosheath by the Cluster spacecraft, Nonlinear Process Geophys., 10, 3-11.

Pickett, J. S., et al. (2004), Solitary waves observed in the auroral zone: The Cluster multi-spacecraft perspective, Nonlinear Process Geophys., 11, 183-196.

Pickett, J. S., et al. (2005), On the generation of solitary waves observed by Cluster in the near-Earth magnetosheath, Nonlinear Process Geophys., 12, 181-193.

Pickett, J. S., et al. (2007), Propagation of electrostatic solitary waves in the magnetosheath: multi-spacecraft observations and simulations, Geophys. Res. Abstr., 9, 03106.

Pritchett, P. L. (2001), Geospace Environment Modeling magnetic reconnection challenge: Simulations with a full particle electromagneticcode, J. Geophys. Res., 106(A3), 3783-3798, doi:10.1029/1999JA001006.

Retińo, A., et al. (2006), Structure of the separatrix region close to a magnetic reconnection X-line: Cluster observations, Geophys. Res. Lett., 33, L06101, doi:10.1029/2005GL024650.

Runov, A., et al. (2003), Current sheet structure near magnetic X-line observed by Cluster, Geophys. Res. Lett., 30(11), 1579, doi:10.1029/2002GL016730. Sergeev, V., et al. (2003), Current sheet flapping motion and structure observed by Cluster, Geophys. Res. Lett., 30(6), 1327, doi:10.1029/ 2002GL016500

Shin, K., H. Kojima, H. Matsumoto, and T. Mukai (2008), Characteristics of electrostatic solitary waves in the Earth's foreshock region: Geotail observations, J. Geophys. Res., 113, A03101, doi:10.1029/2007JA012344.

Wang, R., Q. Lu, X. Li, C. Huang, and S. Wang (2010), Observations of energetic electrons up to $200 \mathrm{keV}$ associated with a secondary island near the center of an ion diffusion region: A Cluster case study, J. Geophys. Res., 115, A11201, doi:10.1029/2010JA015473. 